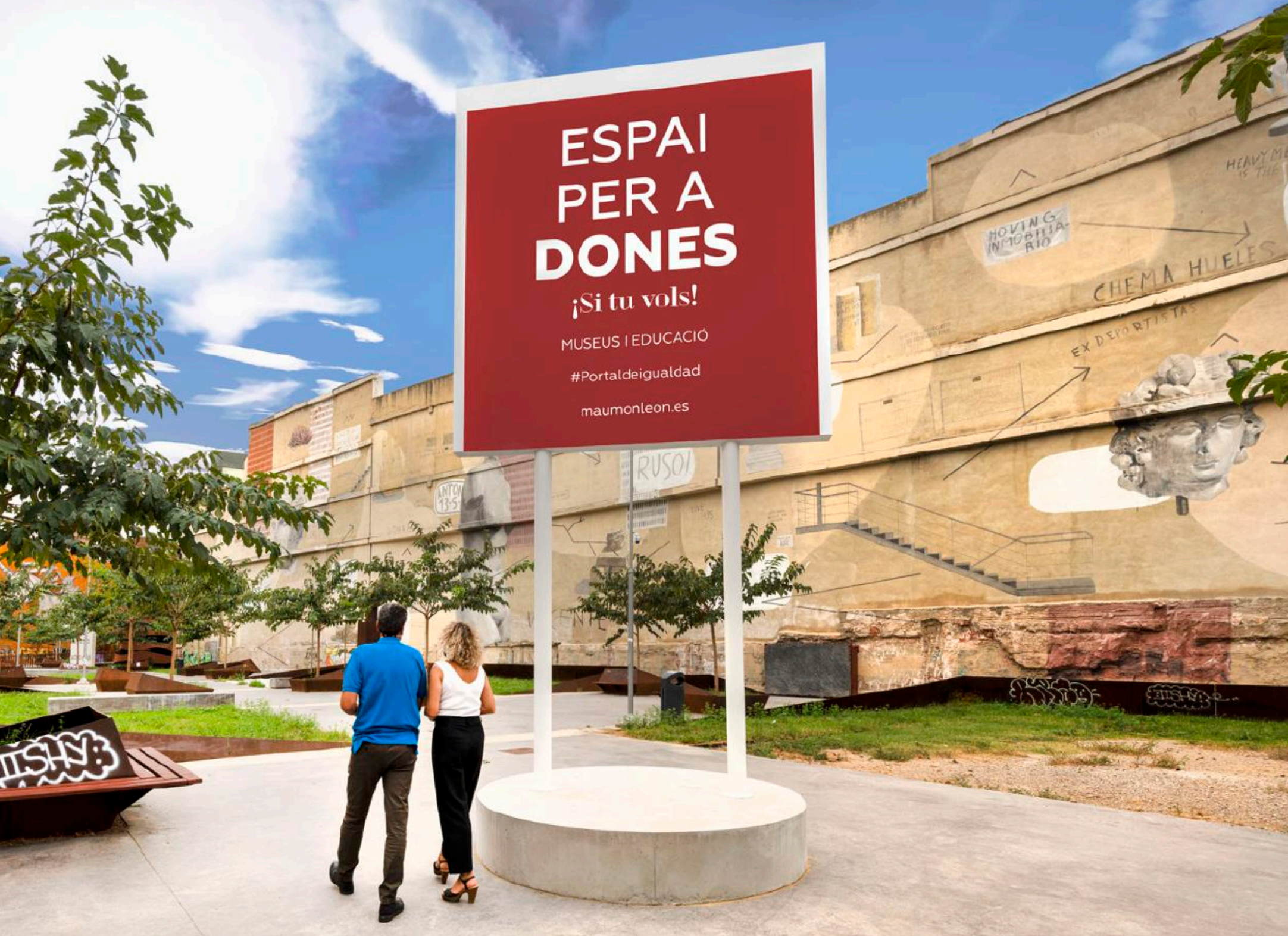

Mau Monleón Pradas, \#Portaldeigualdad / Campaña por la Igualdad en el Museo y en la Educación, 2020-2021. Arte público participativo. Valla publicitaria, Jardín de Esculturas del Instituto Valenciano de Arte Moderno, IVAM. Creado para el proyecto «Portal de Igualdad, IVAM produce» del ivam. Fotografía: Juan García Rosell. 


\section{MUSEOS EN IGUALDAD}

\section{MUSEUMS IN EQUALITY}

\section{Amparo Zacarés Pamblanco Colectiva Portal de Igualdad}

Resumen En su origen los museos respondieron a la cultura de su época y a la lógica patriarcal imperante que dejaba sin visibilidad las obras de las mujeres artistas. Una situación de desigualdad que se prolongó durante siglos y que hizo que la contribución de las mujeres a la cultura y a las artes no fuera lo suficientemente conocida. Ante esto se hace preciso analizar el componente ideológico que ha dominado la historia universal del arte y que sigue obstaculizando las condiciones de distribución y exposición de las obras de las mujeres artistas en los museos, en las galerías y en los centros de arte. En este contexto, cabe destacar la participación de la campaña de arte público trasmedia \#PortaldeIgualdad y la creación de la Herramienta MAV de Autodiagnóstico para la Igualdad en Museos y Centros de Arte en la que se demanda la perspectiva de género en la gestión museística como ejemplo de buenas práticas museísticas y de una cultura auténticamente democrática.

Palabras clave Museos, igualdad, canon androcéntrico, lógica patriarcal, historia del arte.

Abstract IMuseums originally reflected the culture of their age and a dominant patriarchal logic that deprived female artists of recognition and visibility. These were times when the aesthetic cannon followed androcentric patterns, being the qualities of genius attributed only to men. Such inequality, perpetuated over the centuries, rendered the contributions of women to art and culture insufficiently known. For this reason, it is necessary to analyze the ideological component that has long dominated world art history and still hampers the conditions in which female artists distribute and exhibit their works in museums, galleries, and art centers. In this context, it is worth mentioning the participation of the trans-media public art campaign \#EqualityPortal and the creation of the MAV Tool for Self-Diagnosis for Equality in Museums and Art Centers in which the gender perspective is required in museum management as an example of good practice and of a truly democratic culture.

Keywords Museums, Equality, Androcentric Cannon, Patriarchal Logic, Art History. 
En sus inicios los museos asumieron la lógica patriarcal dominante que dejaba sin valor las obras de autoría femenina al considerar que las mujeres no eran sujeto creador. Debido a esto, el goce estético quedaba limitado a las creaciones que se podían contemplar en sus estancias y que custodiaban, por norma general, obras realizadas por varones. De tal modo que los museos, concebidos tradicionalmente como lugares de alta cultura, trasmitieron durante siglos un discurso expositivo que invisibilizaba la obra de las mujeres artistas y las dejaba sin reconocimiento. Pero ¿cómo iban a ser conocidas, si fueron ocultadas, silenciadas y hasta borradas? Como mucho, algunas más destacadas y de épocas más recientes, fueron nombradas y citadas en unas pocas líneas en algunas enciclopedias de arte. Por este motivo, plantear un cambio de paradigma cultural en la política museística supone adentrarnos en sus precedentes, analizarlos e intentar cambiarlos. De ahí que no esté de más recordar que, en sus inicios, los museos fueron un lugar donde se conservaban y exponían las obras de arte que procedían sobre todo de colecciones regias o aristocráticas. Pero a finales del siglo XVIII, en medio de un ambiente revolucionario que provocó la caída de las monarquías, estas colecciones se abrieron al público y pasaron posteriormente a formar parte de los primeros fondos museísticos. Eran tiempos en los que el canon estético seguía patrones androcéntricos y el concepto de genialidad solo era atribuido al varón. Es más, los criterios estéticos los decidían las academias y en ellas no había cabida para las mujeres artistas.

Fue en 1792 cuando se creó en el palacio del Louvre el primer museo de arte y a este le siguieron otros más. De hecho, los grandes museos se desarrollaron en Europa en el siglo xIX, aunque no sería hasta las primeras décadas del siglo $\mathrm{xx}$ cuando comenzaron a estructurarse de forma científica. En esos años aparecieron también los primeros museos de arte contem- poráneo como el Museum of Modern Art fundado en 1929 y construido en Nueva York en 1939. Más o menos en la misma línea, desde entonces y según sus posibilidades, los fondos documentales y las colecciones de los museos contemporáneos se caracterizan por contar con obras emblemáticas de grandes maestros que ejemplifican algunas de las vanguardias y movimientos del arte contemporáneo. Es pues un hecho constatado que, en general, en los fondos museístos hay poca obra realizada por mujeres artistas. Sin embargo, en la actualidad puede decirse que el viento es más favorable y que ya se vislumbra una nueva sensibilidad. Debido a ello, el paradigma androcéntrico en el arte comienza a agrietarse y hundirse. En otras palabras, ya nada puede justificar la injusticia cometida al excluir la contribución de las mujeres en la historia del arte, máxime cuando una de las prioridades de la política cultural de nuestra época es la democratización de los museos, de los centros culturales y de las instituciones artísticas.

\section{I}

En realidad, solo una visión banal de la historia del arte puede restar hoy protagonismo a las mujeres como creadoras, convirtiéndolas en meras musas o modelos de los artistas varones. Ya no se sostiene esa visión sesgada, sobre todo desde que en los años setenta del siglo anterior al nuestro, tomaron impulso las investigaciones dedicadas a rescatar del olvido a las artistas del pasado que, siendo valiosas y con talento, no fueron reconocidas en su valía. Eran artistas, por decirlo de manera sintética, «ilustres y desconocidas» (Arribas, 2007). En este contexto, fue determinante el artículo que publicó en 1971 Linda Nochlin y que hoy es todo un referente de la crítica feminista. En aquel texto, la autora se preguntaba: «¿Por qué no ha habido grandes mujeres artistas?» Es obvio que la respuesta incidía en la discriminación estruc- 
tural que sufrían las mujeres dentro de un paradigma cultural que no atribuía ni ingenio ni talento a sus creaciones artísticas. Desde luego el concepto de genialidad creativa vinculado al éxito comercial no era propio de las mujeres. Un hecho que no pasó inadvertido a las Guerrilla Girls, tal como lo denunciaron en su «Código ético para Museos de Arte» (1985). Se trataba de un decálogo de corte feminista y antirracista, cuyo décimo mandamiento decía en tono de burla vagamente disimulado: «Admitirás ante el público que términos como genio, obra maestra, seminal, potente, duro, enérgico y poderoso, tan sólo se utilizan para apoyar el mito e inflar el valor de mercado de los artistas blancos varones».

Unas palabras que no han perdido actualidad puesto que, en estos momentos, aun dándose algunos avances, la situación no ha cambiado en demasía. De hecho, las Guerrilla Girls, en el texto que publicaron en 1988 y que titularon "Las ventajas de ser mujer artista», reunieron las mismas denuncias que hoy podrían hacer muchas de las mujeres artistas de este nuevo milenio. Muchas, sin duda, pueden verse reflejadas en este escrito en el que las activistas ironizaban sobre las ventajas de ser mujer y artista. Entre estas ventajas estaba: «Trabajar sin la presión del éxito»; «No tener que coincidir con hombres en las exposiciones»; "Saber que tu carrera profesional puede repuntar cumplidos los ochenta años»; "Tener la garantía de que, no importa el tipo de arte que hagas, se etiquetará siempre como femenino»; "Ver tus ideas perpetuadas en las obras de los demás»; «Tener la oportunidad de elegir entre una carrera profesional y la maternidad»; "Que te incluyan en versiones revisadas de la historia del arte»; «No tener que pasar por el bochorno de que te llamen genio»; o «Que tu imagen salga en las revistas de arte disfrazada de gorila» -en una clara referencia a sí mismas-.

Dicho esto, hay que reconocer que las cuestiones relativas a la invisibilidad de las mujeres en la historia y en el mercado del arte, no es algo reciente ni novedoso. El predominio durante siglos del androcentrismo que privilegia al varón y lo masculino en cualquier campo de la cultura, de la política y del saber, hizo evidente que ser mujer y artista llevaba un plus añadido de dificultad que no se tenía en cuenta. De ahí la importancia que tuvo el activismo de las Guerrilla Girls cuando, a mediados de los ochenta del siglo pasado, aquellas mujeres que portaban máscaras de gorilas para esconder su identidad, denunciaron la discriminación que las artistas sufrían en los museos y en las galerías de arte. Con sus acciones evidenciaron que, en las galerías comerciales más conocidas de Nueva York, menos del 10\% de las obras eran de mujeres artistas. También visibilizaron que ninguna mujer artista había realizado una exposición individual en el Guggenheim, en el Metropolitan o en el Whitney y que solo una había expuesto en la Tate Modern. Fueron las primeras activistas en revelar que, durante siglos, los museos habían estado llenos de arte hecho por hombres como si no hubiera habido mujeres artistas que pudieran merecerlo.

Es de reseñar que su activismo no se agotó en aquellas décadas del siglo pasado y todavía siguen estando en activo colaborando a nivel internacional en los proyectos a los que se les invita. Al respecto, es interesante recordar el trabajo artístico que las Guerrilla Girls realizaron junto a Valentina Paz Enríquez, Dionisio Sánchez y Mau Monleón Pradas (2017: 13). De resultas de esta colaboración, se realizó en la Universidad Politécnica de Valencia la obra colectiva Museum Recount Spain 2010/2013, dentro de la exposición «Women in Work. Mujer, Arte y Trabajo en la Globalización». En esta ocasión, se tomó como fuente el informe MAV sobre la desigualdad de género en las artes y se mostró, con la tipografía visual que les caracteriza, el número de hombres y mujeres que participaron en las exposiciones indivi- 


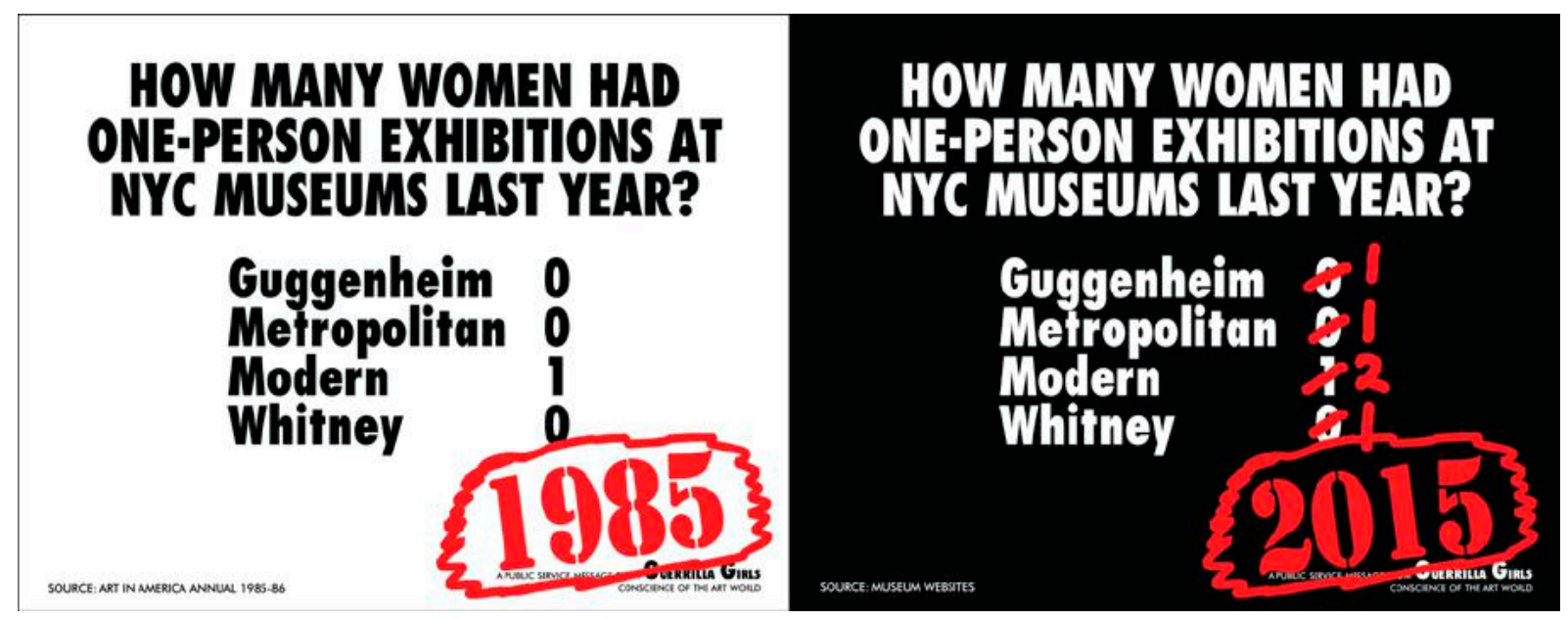

Guerrilla Girls. Museum Recount 1985/ 2015.

\section{HOMBRES/MUJERES

$\begin{array}{lrr}\text { IVAM } & 61 & 9 \\ \text { MACBA } & 18 & 4 \\ \text { MNCARS } & 37 & 14 \\ \text { CAC } & 32 & 7\end{array}$

EXPOSICIONES INDIVIDUALES EN CENTROS DE ARTE DE ESPAÑA ENTRE LOS AÑOS 2010-2013:

duales que tuvieron lugar entre los años 2010 y 2013. Las cifras constataron la desigualdad de género y la falta de equidad que existía en la gestión cultural, en este caso en varios centros de referencia de arte contemporáneo como son el IVAM, MACBA, MNCARS y CAC.

\section{II}

Llegados a este punto, no queda otra posibilidad que analizar el componente ideológico que ha dominado la historia universal del arte y que sigue obstaculizando las condiciones de distribución y exposición de las obras realizadas por mujeres. Quizás pueda parecer lejana o trasnochada esa mentalidad incrédula que niega que obras de gran calidad artística puedan salir de la imaginación y de la mano de una mujer. Pero no lo es, aún se observan algunas apreciaciones de este tipo que buscan desprestigiar a las mujeres artistas. Como sucedió, apenas hace unos años, cuando el pintor alemán Georg Baselitz, en unas declaraciones al semanario Der Spiegel en 2013, sostenía que las creaciones de las mujeres son obras menores, que no dan la talla y que, sal- 


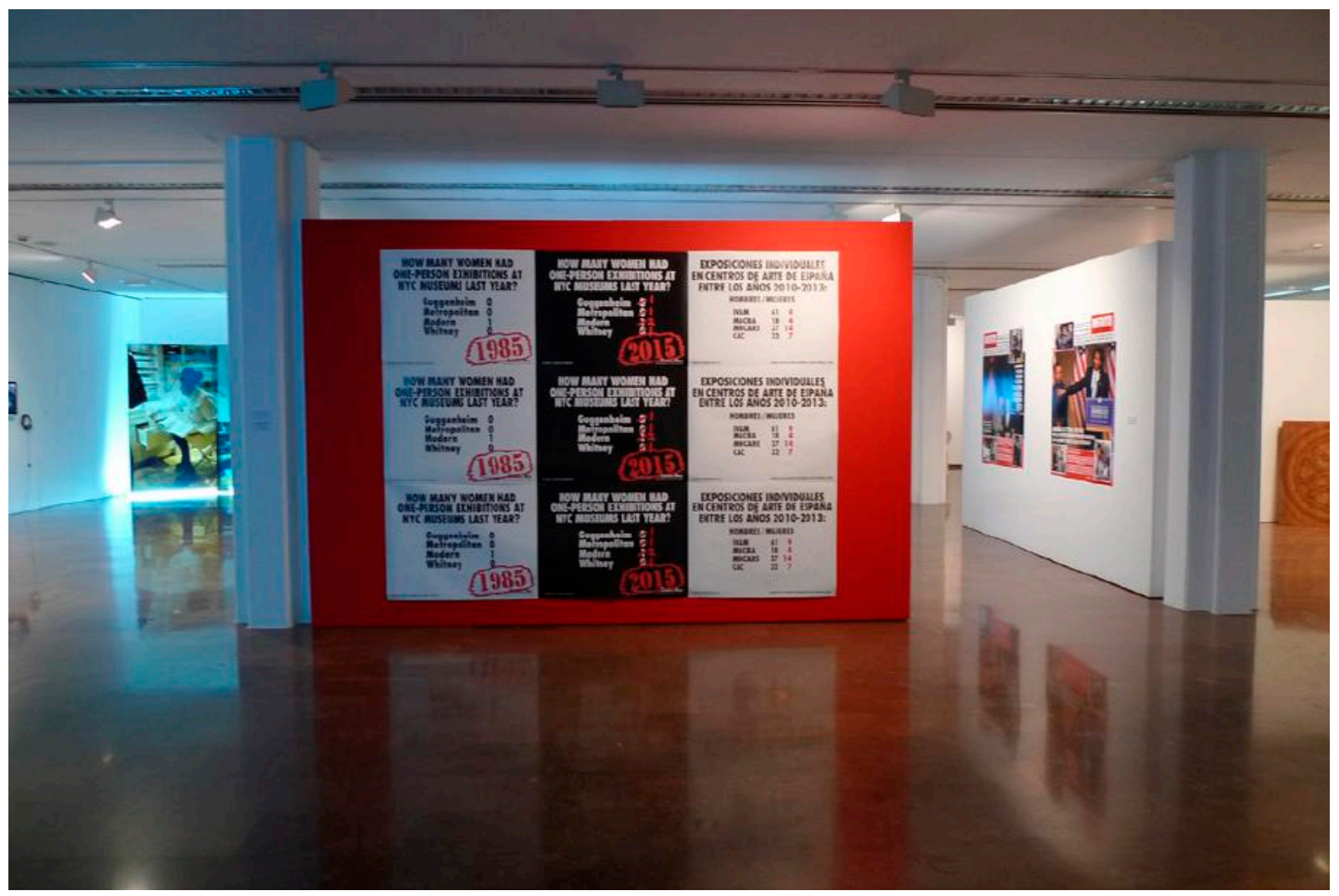

Guerrilla Girls en colaboración con Valentina Paz Enríquez, Dionisio Sánchez y Mau Monleón Pradas. Women in work. Mujer arte y trabajo en la globalización. Universidad Politécnica de Valencia, 2017.

vo alguna excepción, merecen poca consideración y mención. Unas palabras con las que alimentaba el mito del universalismo masculino en la cultura y bendecía el mercado del arte hecho por y para los varones artistas. De todos modos, a estas alturas del devenir histórico, tales aseveraciones de corte misógino se rechazaron de inmediato con datos contrastados; así, mientras que Der Spiegel reproducía las palabras de Baselitz, otro periódico, The Independent, las cuestionaba dando cifras de las artistas que sí son valoradas en el mercado del arte. Desde luego, las apreciaciones negativas hacia las mujeres artistas no son de recibo cuando se sabe que su ausencia en el sector de las artes no responde necesariamente a criterios de calidad sino a circunstancias ligadas al techo de cristal, a la brecha salarial o al hecho de que sean sobre todo varones los coleccio- nistas y los directores de museos y ferias de arte. Ante estas evidencias, no resulta extraño que en la agenda actual del feminismo esté anotado reivindicar que las mujeres queden proporcionalmente representadas para que se modifique sustancialmente el panorama de su invisibilidad en los espacios del arte y de la cultura en general.

Con todo, es un hecho que el mercado del arte se perpetúa en masculino. Por este motivo, hay que tomar postura ante la desigualdad estructural que los sexos han arrastrado históricamente y ser conscientes del silenciamiento de la participación femenina en la historia del arte. Una injusticia que, desde el asociacionismo de mujeres, se ha querido denunciar y modificar. En el ámbito estatal, por ejemplo, la asociación interprofesional de Mujeres en las Artes Visuales (MAV) organiza desde hace años 

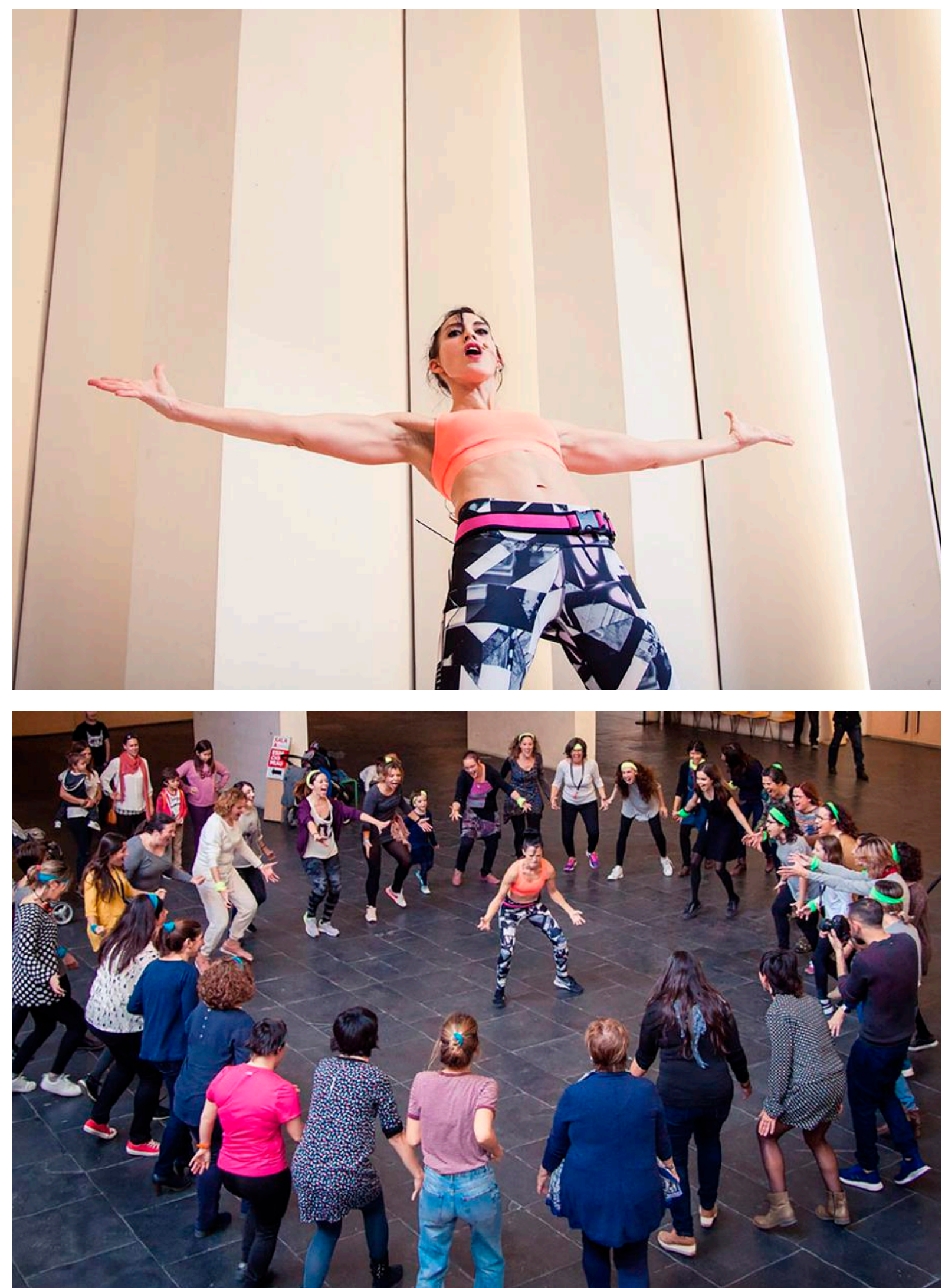

Acción participativa de Yolanda Domínguez. Gym Win Session. Feria Marte, Castelló, 2017. Fotografías: Marcos Almuedo. 

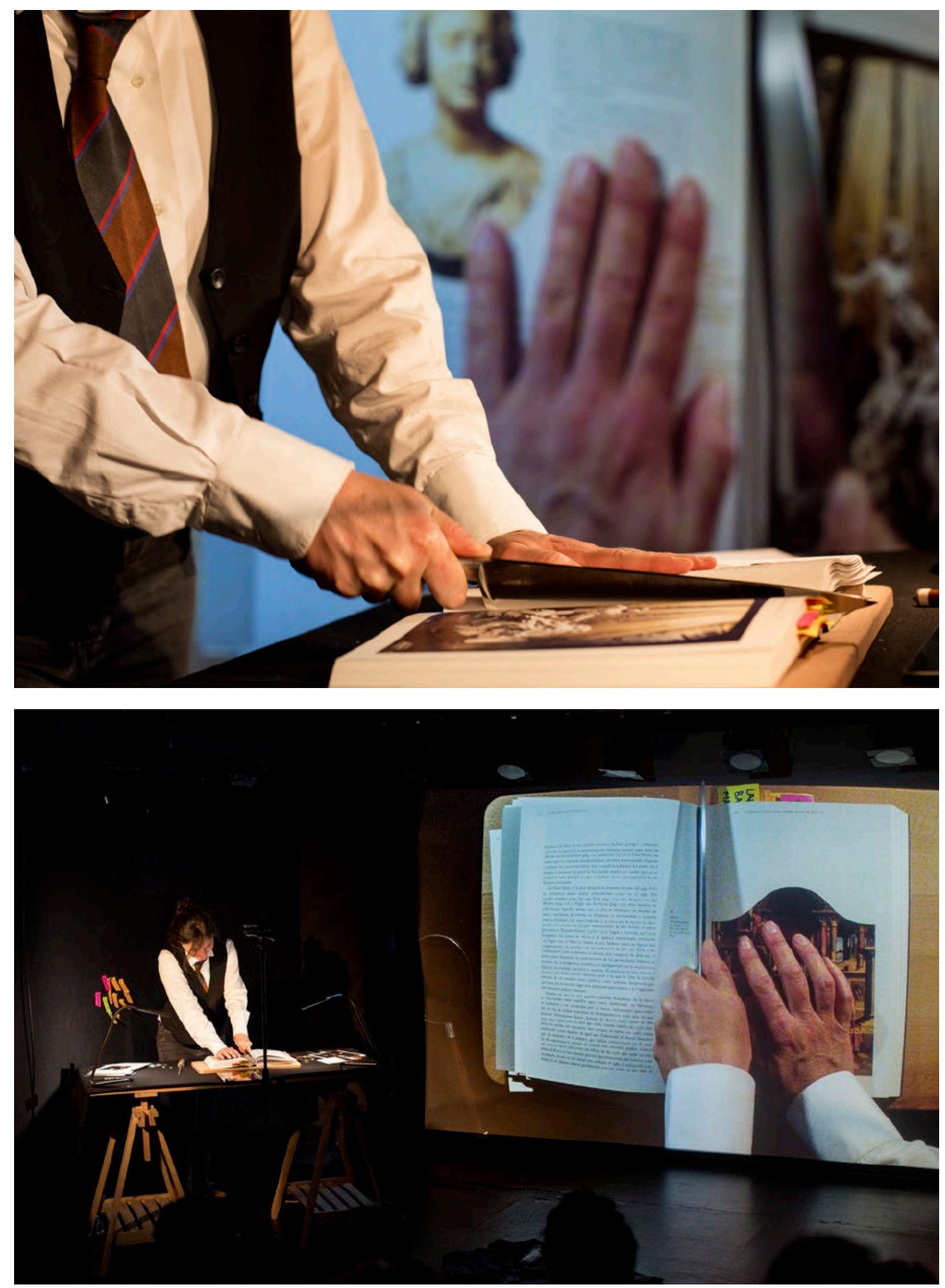

La artista María Gimeno realiza su performance Queridas viejas. Teatro Off Latina de Madrid, 2018. Fotografías: Pedro Martínez Albornoz. 


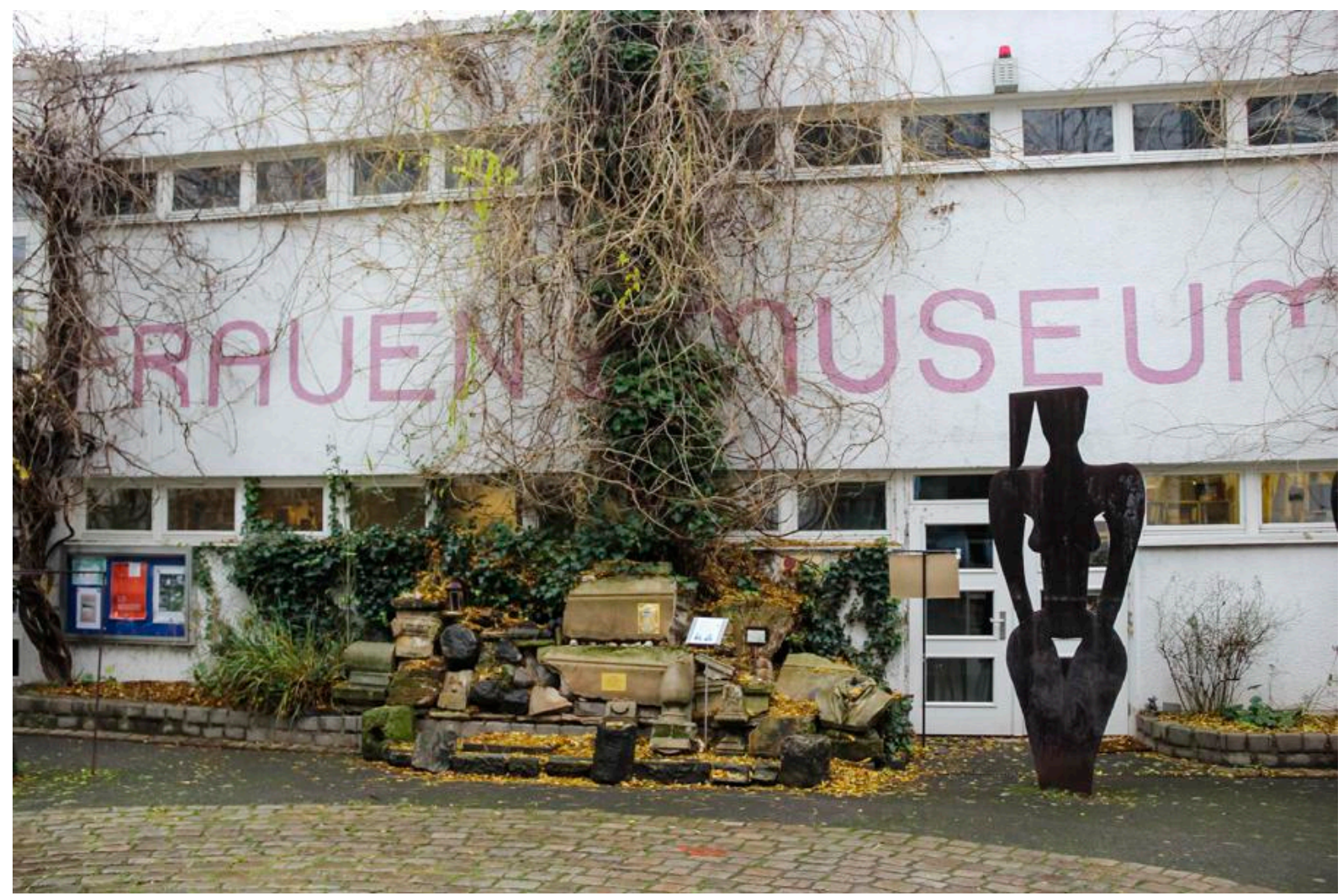

Frauenmuseum. Bonn. Gertrudiskapelle. @ Axel Kirch / CC BY-SA 4.0 (Wikimedia Commons)

https//commons.wikipedia,org/wiki/File:2017-12-18-Bonn- Frauenmuseum-Gertrudiskapelle-06.jpg

el Festival de Miradas de Mujeres para que estas puedan exponer y presentar sus obras. A tal efecto resultan muy reveladoras acciones participativas como \#estamosaquí, de la artista multidisplinar Yolanda Domínguez, realizada en ARCo en 2018, en la que las mujeres artistas se geolocalizaron y recorrieron las instalaciones de la feria para denunciar el machismo imperante, una reivindicación en la que persiste y en la que no está sola. Otra de sus acciones, realizada en la Feria Marte 2017, hablaba de fuerza y empodeamiento (Feliu, 2019).

También en la dirección de la visiblilidad, se sitúa la obra de la artista multidisplinar contemporánea María Gimeno, quien cuestionando a Gombrich -toda una autoridad en la crítica canónica de arte- reivindica de forma enérgica y vehemente el lugar de las mujeres artistas en la historia del arte. Con esa finalidad creó su con- ferencia performativa titulada «Queridas Viejas Project», que ha tenido una gran acogida y difusión en las redes. En la acción rehace el libro canónico de E.H. Gombrich sobre historia del arte incluyendo en él a las mujeres creadoras con las que no se contó.

Puestas así las cosas, conviene recalcar la situación excepcional en la que en este año 2021 se ha celebrado la Feria Internacional de Arte Contemporáneo ARComadrid, por primera vez en julio, por razones obvias de la pandemia. Por el momento, aún no se disponen de datos de la participación de las mujeres artistas en ese recinto, pero sí se sabe que ha sido una edición en la que han participado 130 galerías y algo más de 1500 artistas. Cabe destacar que, en esta ocasión, se ha fomentado la presencia de las artistas, solicitando a las galerías que en los «Proyectos de Artistas», espacios diferencia- 


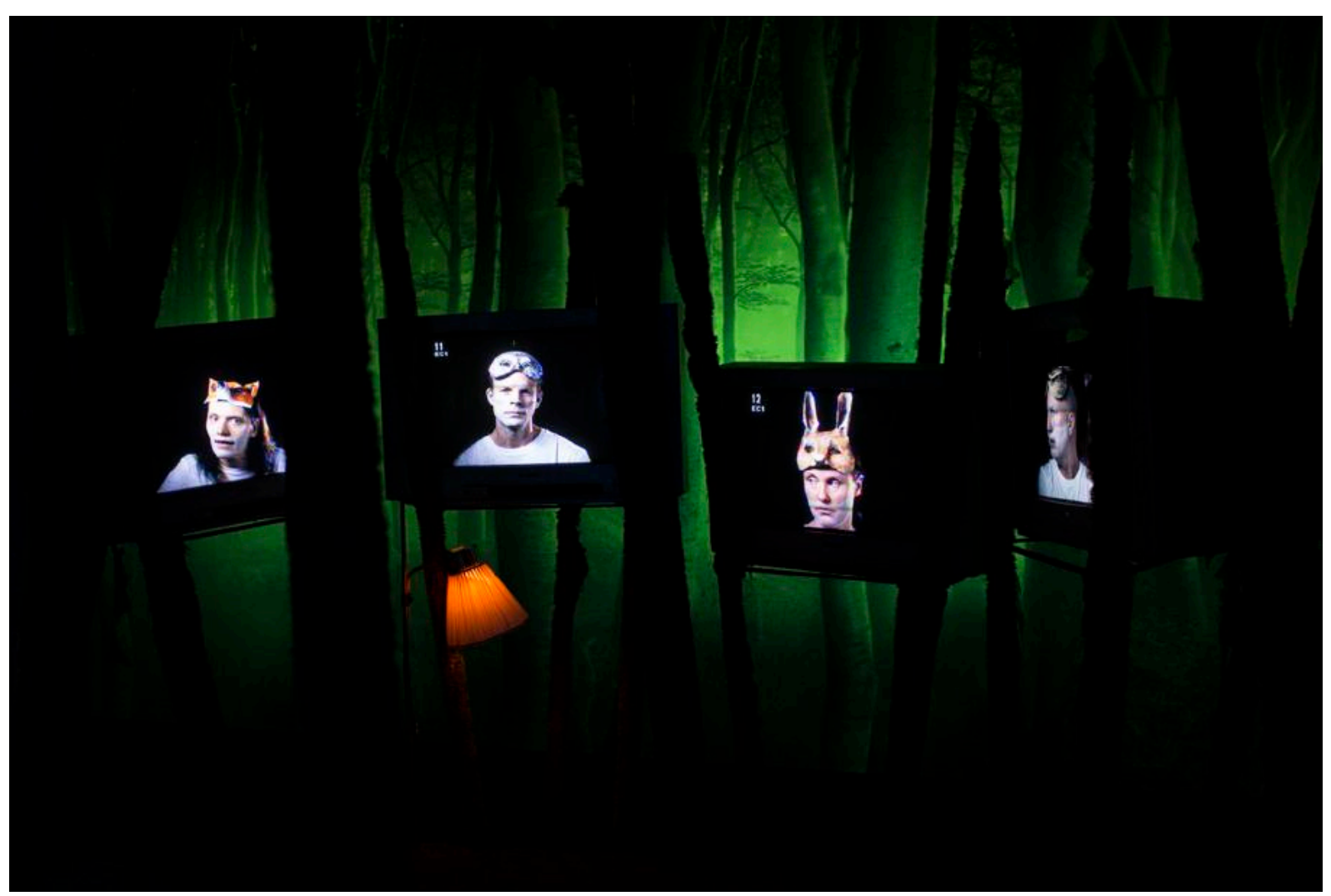

Exposición Roots - a Tangled History. A dark story about gender \& power, Identity \& history Kvinnohistoriskt museum, Umeå (Suecia). @ Malin Grönborg (Wikimedia Commons)

https://upload.wikimedia.org/wikipedia/commons/e/ea/The_exhibition_Roots_a_Tangled_History_at_the_Museum_of_Womens_History.jpg

dos dentro de la feria donde se exhiben obras individuales, se expusieran solamente obras de autoría femenina. Sin embargo, al no contar con alguna estadística contrastable, no se puede decir con seguridad en qué grado la situación ha variado o no. Ahora bien, dado que los cambios sustanciales no se producen de la noche a la mañana, lo más probable es que se siga manteniendo el porcentaje mínimo que ya se reveló en la edición pasada, gracias al informe MAV 2020. Un documento que desvela que el porcentaje apenas se ha movido, manteniéndose en un escaso $7 \%$, una dinámica que ha sido, más o menos, la habitual en estos diez últimos años.

Por otra parte, el hecho de potenciar a las artistas en un espacio diferenciado, no parece ser la estrategia más acertada para reivindicar la igualdad de trato y de reconocimiento, ya que separa a las mujeres artistas del conjunto de la producción artística y presenta sus obras como si fueran algo marginal e inusual. Sea como sea, la cuestión es que aún no se da la paridad y las cifras que se manejan lo evidencian con total claridad. Así lo reflejaba el Informe que Aernet publicó en 2019, donde la representación de artistas femeninas era solo un $2 \%$. Datos que dejaban claro que poco o nada había cambiado. En esa línea, no resulta extraño que Peio H. Riaño (2021) recordara en prensa que, según el estudio de mercado de 2020 publicado por Art Basel y uBs, la proporción de las mujeres artistas representadas por galeristas todavía era minoría. Con ello, dejaba en evidencia el retroceso hacia la equidad que se está produciendo, ya que mientras en 2019 el porcentaje de mujeres artistas fue de $41 \%$, en el 2020 , año de la pandemia, fue 
solo de $39 \%$. Una situación injusta que hay que abordar y desarticular.

En ese sentido, contar y extraer porcentajes es una operación muy efectiva para mostrar el estado de la situación. Los resultados nos indican dónde estuvimos, dónde estamos y dónde queremos estar. Es un procedimiento mecánico que, como se ha señalado antes, ya hicieron las Guerrilla Girls a mediados de los años ochenta del siglo pasado y que se sigue haciendo hoy para recabar datos objetivos. De tal modo que, ante la ausencia de paridad y de igualdad de oportunidades, la presencia de las mujeres artistas en los museos y centros de arte se presenta hoy como una cuestión candente que no puede ser ignorada ni desatendida.

\section{III}

Estas consideraciones previas han servido para abordar la temática que nos ocupa con mayor propiedad. De algún modo ofrecen la fotografía de una problemática que no hay que perder de vista para tomar conciencia de la exclusión de las mujeres en el arte como sujetos de creación. Una situación que en gran parte se produce porque los puestos directivos de la industria cultural los suelen detentar los varones. Si a esto se añade que para entrar en el mercado del arte hay que tener visibilidad y que la trayectoria de una carrera artística viene determinada, en gran medida, por haber colaborado con las galerías que participan asiduamente en las ferias internacionales, queda patente la urgencia de una política cultural que defienda acciones positivas para lograr la paridad en el arte.

En este caldo de cultivo, a finales del siglo pasado, los discursos programáticos dieron paso a la acción y ya es un hecho manifiesto la existencia de varios museos de mujeres. Como, por ejemplo, el National Museum of Women in the Arts (NMWA) de Washington D.C., creado en 1981 o el Frauenmuseum de Bonn (Alema- nia) cuya creación data también de ese mismo año. Más reciente se creó el Frauenmuseum de Hittisau en Austria, fundado en 2000 y dirigido por Stefania Pitscheider. En todos estos museos la meta es reconocer los logros artísticos y culturales de las mujeres. En concreto, en el museo austríaco se destacan sobre todo las aportaciones artísticas de las mujeres en el ámbito local y su programación suele contener diversas actividades y talleres didácticos para público general e infantil. Con todo, lo que importa es enfatizar que el Frauenmuseum Hittisau nace en los albores de este segundo milenio y que ha sido distinguido con el Premio del Museo Austriaco 2017, un distintivo que dice mucho de la consideración y el valor que su país da a la potencialidad creativa con autoría femenina. Es más, este museo pertenece a la International Association of Women's Museums (IAWM) fundada en 2012 por Alice Springs. En realidad, la génesis de esta asociación se gestó años antes, ya que surgió de la red de Museos de la Mujer que se fundó en Merano (Italia) en 2008. En cualquier caso, lo importante es que se trata de una asociación cuyo objetivo es vincular a todos los museos de las mujeres que en estos últimos años han proliferado por todo el mundo. Además hay que destacar que la IAWM supuso un punto de inflexión para el asunto que nos ocupa, puesto que presenta la tipología de Museos de la $\mathrm{Mu}-$ jer como espacios de resistencia ante un pensamiento hegemónico excluyente.

Podemos decir que estos museos fueron la respuesta a la necesidad de revisar la transmisión de la historia como proceso en el que la norma era el silenciamiento de la contribución de las mujeres a la cultura. A decir de Marián López Fernández-Cao, en la búsqueda hacia la equidad se hace preciso asumir que «las mujeres y los hombres de todas las geografías y tiempos han sido sujetos creadores dentro de la historia y la crítica de arte» (2008: 94). De ahí el imperativo de «deconstruir las dicotomías 


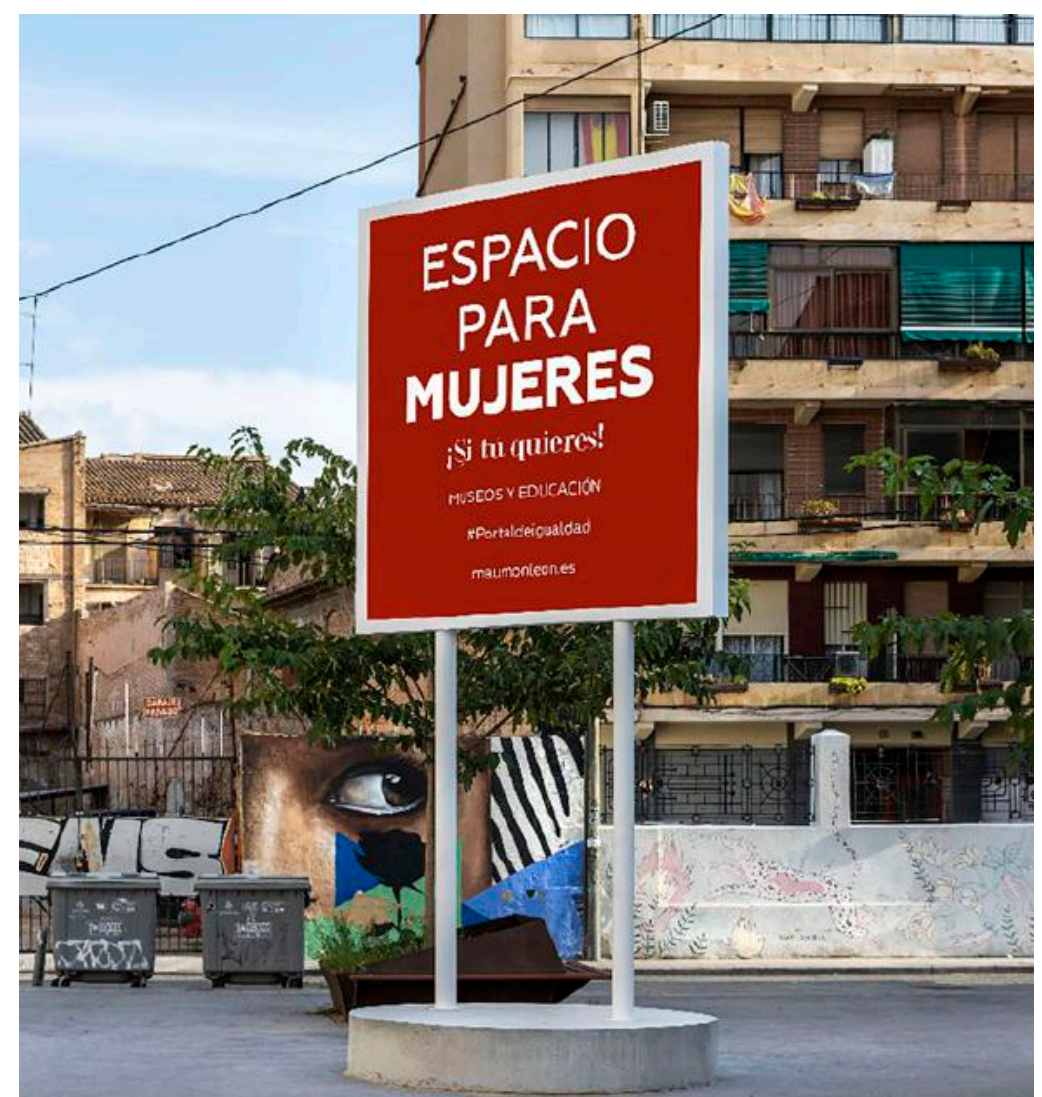

Mau Monleón Pradas, \#PortaldeIgualdad / Campaña por la Igualdad en el Museo y en la Educación, 2020-2021. Arte público participativo. Valla publicitaria, Jardín de Esculturas del Instituto Valenciano de Arte Moderno Ivam. Fotografía: Juan García Rosell. Creado para el proyecto «Portal de Igualdad, IVAM produce» del IVAM.

duales excluyentes y resignificarlas». Una consideración que estuvo en la base de los museos citados, al igual que en el National Women's History Museums (NMwA), fundado por Karen Staser en 1996, un museo virtual que lleva tras de sí mucha trayectoria y cuya sede actual se encuentra dentro del Washington Smithsonian Institute. En ese sentido, aunque en una línea marcadamente feminista que lo diferencia de los anteriores Museos de la Mujer, puede citarse también el Kvinnohistoriskt Museum de Umea (Suecia), que se fundó en 2014 y que está en la actualidad dirigido por Maria Perstedt. Ante estas evidencias puede decirse que, con mayor o menor intensidad, ha ido tomando peso un proceso de deconstrucción y de resignificación que incorpora nuevas vo- ces y otras miradas en los museos. En consecuencia, la gestión museística se ha hecho más crítica y ha asumido nuevos parámetros para enfocar el arte y la cultura con equidad.

\section{IV}

No en vano el momento actual resulta más propicio que nunca para impulsar una gestión cultural comprometida con la igualdad de forma real y efectiva. En ese compromiso que tiene como referencia jurídica la Lo 3/2007 para la Igualdad efectiva entre Mujeres y Hombres, se sitúan algunas propuestas que recientemente han aparecido en el panorama nacional pero que también se postulan para aplicarse a nivel internacional. Se trata de diversas iniciativas que in- 
corporan la perspectiva de género interseccional como ejemplo de buenas prácticas museísticas y de una cultura auténticamente democrática. En apenas un año esas directrices se canalizaron por varias fuentes. Una de ellas partió de $\mathrm{Mu}$ jeres en las Artes Visuales (MAV) y se presentó en febrero de 2020 como Herramienta MAV de Autodiagnóstico para la Igualdad en Museos y Centros de Arte. El documento explicativo ${ }^{1}$ contiene su fundamentación teórica y metodológica (López Fernández-Cao y Porta Lledó, 2020), así como un análisis de los museos como espacios de educación y des/legitimación política y social. Así se señala que:

Los museos son espacios de autoridad singulares que marcan un lugar de significación y trascendencia. Son escenarios donde acontece lo importante en términos culturales, donde cristaliza lo más valioso de cada cultura. Lo que debe permanecer. El museo, al presentarnos de modo solemne piezas elegidas del pasado, educa irreversiblemente, sea la educación un elemento presente o ausente de las programaciones de estas instituciones. Educa desde la presencia y desde la ausencia, desde lo valioso y desde lo desechable, desde lo que considera central a lo que considera anecdótico, desde lo que señala necesario y que define como contingente, desde los fines prioritarios e irrenunciables a los secundarios y suprimibles (2020: 11-12).

A grandes rasgos, el papel socializador y generador de cultura sobresale como característica de los museos actuales. De tal modo que sus funciones son, en esencia, de tipo social, educativo e interpretativo. Ya no se limitan, como sucedía en los museos tradicionales, a buscar la contemplación y el goce estético de las obras de arte, sino que muestran sus fondos museísticos

1 El documento lo firman Marián López Fernández-Cao y Alma Porta Lledó (2020), junto a otras compañeras que participaron en la investigación como grupo de trabajo MAv. Las componentes del grupo de trabajo MAV que participaron en la redacción del documento fueron: Txaro Arrazola, Vanesa Cejudo, Lola Díaz González-Blanco, María José Magaña Clemente y Tonia Fernández Trujillo. o sus exposiciones temporales como un recurso para que el público pueda crear su propio conocimiento. En suma, en comunicación dialogante con el público, se considera a los visitantes del museo como «participantes del proceso de construcción del conocimiento» (Roberts, 1997: 65). De ahí que el museo ya no sea un lugar de saber disciplinario y de alta cultura, sino más bien un «lugar de duda, de controversia y de democracia cultural» (Padró, 2003: 57). En resumidas cuentas, en su función educativa, el museo ofrece una manera de ver el mundo e impulsa la interiorización de una serie de valores que guíen nuestro comportamiento a la hora de relacionarnos entre sí, a nivel personal, social y cultural.

Por este motivo, los museos ya no pueden desentenderse de la equidad, que ha de ser asumida como un imperativo deontológico de las buenas prácticas museísticas. En esa dirección se creó la citada Herramienta MAV para la Igualdad que ya es una realidad y que consiste en una aplicación ${ }^{2}$, dentro de la web oficial de la asociación, a fin de que los museos que quieran llevar a cabo su autodiagnóstico puedan hacerlo y así comprobar y visualizar en qué medida cumplen o no con los parámetros de igualdad y diversidad que existe en la sociedad. Esta herramienta de autodiagnóstico consta de un cuestionario que, una vez contestado, se responde con una serie de sugerencias y una valoración final MAV para que el Museo o Centro de Arte que lo haya completado, pueda consultarlo y adopte prácticas a favor de la igualdad. Esta herramienta es de fácil utilización, y es muy muy útil para ayudar a los museos a realizar un plan de mejora, tanto a nivel epistemológico como metodológico, para que en sus recintos se incluyan las diversas identidades sociales.

2 https://mav.org.es/herramienta-mav-para-la-igualdad/ [Fecha de consulta $26 / 7 / 21$ ] 


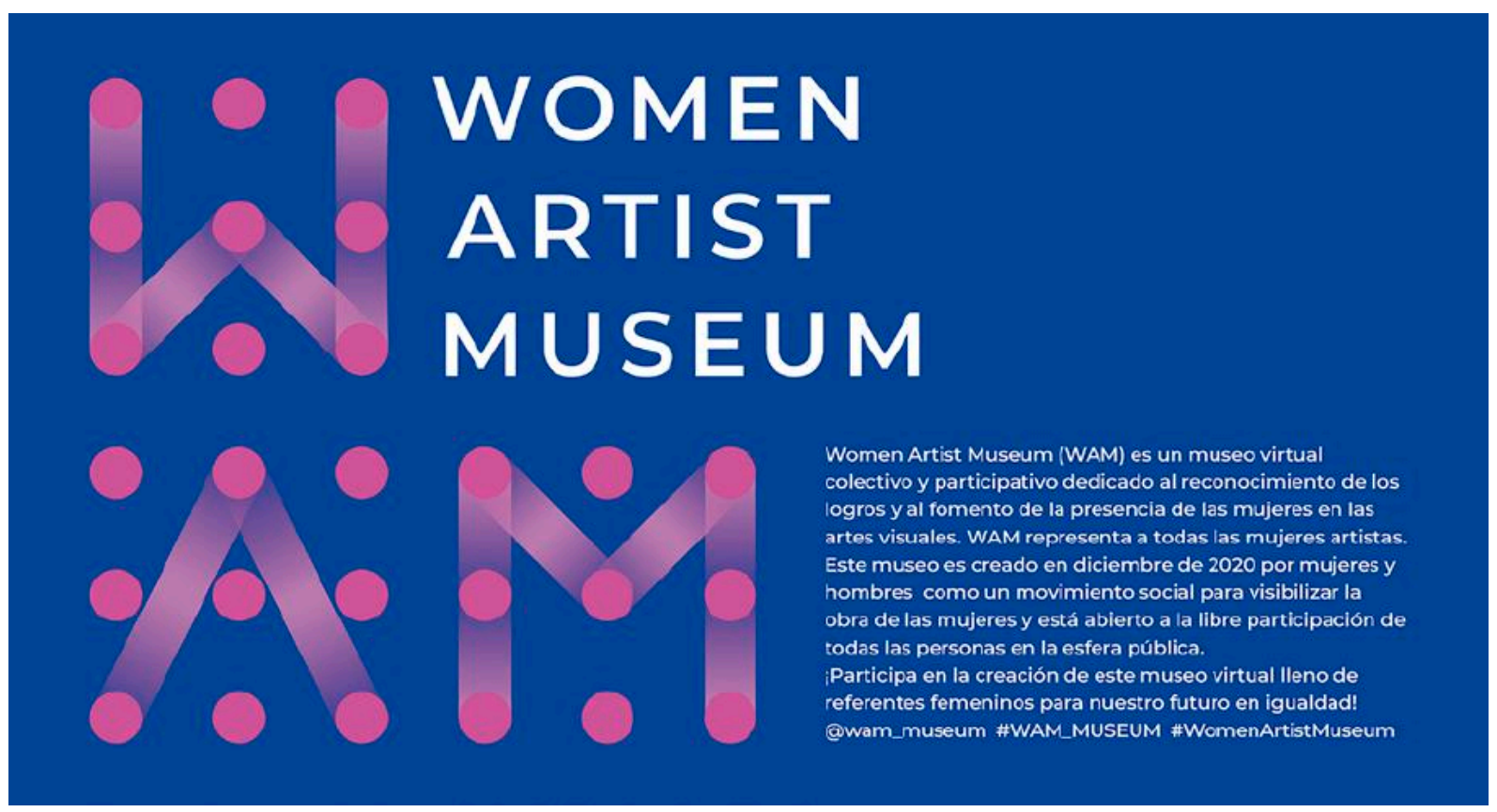

Cartel de la intervención pública en el Ágora del Campus de la UPV ideada por la artista Mau Monleón Pradas con la colaboración de la artista María Penalva-Leal. Correspondiente a la tercera acción de la posteriormente consolidada Colectiva Portal de Igualdad, 29 diciembre 2020.

V

En esa línea, se sitúa la campaña de arte público feminista que la artista multidisplinar Mau Monleón-Pradas creó a instancias del IVAM (Instituto Valenciano de Arte Moderno) a finales de 2020. Como viene a ser característico en su producción artística, se concentró en el proceso de comunicación que se establece entre el público y la obra. En un primer momento, con fecha 7 de septiembre de ese año, lanzó una campaña en redes con el \#PortaldeIgualdad para reclamar la igualdad de oportunidades entre mujeres y hombres en los museos y los centros de arte. Un llamamiento que tuvo su seguimiento y repercusión no solo entre el público en general sino también entre un público especializado del mundo de las artes y de la gestión cultural. Tras este proceso de sensibilización y movilización en redes, creó una valla publicitaria, un cartel donde, sobre fondo rojo con grandes letras en blanco, se leía «ESPACiO PARA MUJERES». A este mismo mensaje, para- fraseando el eslogan de la campaña que difundieron John Lennon y Yoko Ono en 1969 en favor de la paz y contra la guerra de Vietnam, añadió en una tipografía más pequeña la exclamación «iSi tu quieres!». Finalmente la valla publicitaria, con el cartel y sus dos leyendas, se colocó en el entorno del Pati Obert del IVAM y allí estuvo hasta que en marzo de 2021 fue trasladada a la Universidad Politécnica de Valencia para formar parte del parque escultórico de dicha universidad.

En todo este proceso, la intención de la artista fue la de generar una corriente de opinión favorable a un portal de igualdad en las webs de los museos y de los centros de arte como algo no solo deseable sino también urgente. Por ello, apenas un mes después de su lanzamiento, el 29 de octubre de 2020 , se presentó ante la opinión pública el Manifiesto en favor de un Portal de Igualdad en los Museos y Centros de Arte. Este documento, que se redactó colaborativamente, contenía como principio programático dar visibilidad a las aportacio- 
nes de las mujers artistas para que la cultura fuera más justa y democrática. El Manifiesto se leyó en varios idiomas y se difundió en redes solicitando su adhesión, que se firmó tanto a título individual como a nivel institucional por diversas asociaciones y entidades culturales.

Un punto de inflexión en este proyecto de arte público feminista aconteció cuando un grupo de personas procedentes del ámbito de la creación artística, de la docencia universitaria y de la gestión cultural formaron, en torno a la artista Mau Monleón Pradas, la Colectiva Portal de Igualdad ${ }^{3}$ para seguir demandando igualdad de representación y de oportunidades entre mujeres y hombres en los Museos y Centros de Arte. Con esta intención, la Colectiva se reúne regularmente todos los meses para preparar las acciones a realizar y mantener viva la reivindicación del enfoque de género para los espacios de arte. A tal efecto, con fecha 29 de cada mes, rememorando el día que se dio a conocer el Manifiesto Portal de Igualdad, se presentan en prensa las acciones realizadas ese mes.

Entre ellas pueden destacarse, a modo de ejemplo, el Women Artist Museum (WAM), un museo virtual colectivo y participativo dedicado al reconocimiento y al fomento de la presencia de las mujeres en las artes

3 La Colectiva Portal de Igualdad la conforman en su núcleo fundacional: la artista, curadora y editora visual Liliam Amaral (Sao Paulo, Brasil); la artista visual Mar Caldas (Vigo, España); el artista y escritor Samuel Gallástegui (Bilbao, España); la artista y comisaria Celeste Garrido (Pontevedra, España); la artista y comisaria Mau Monleón Pradas (Valencia, España); la artista visual María Penalva-Leal (Alissia) (Valencia, España); la artista y rapsoda Manola Roig (Sueca-Valencia, España); la artista visual Bia Santos (Salvador de Bahía, Brasil); la profesora y directora del Museu d'Art Contemporani de Vilafamés, Rosalía Torrent (Castellón, España); el crítico de arte y gestor cultural Alex Villar (Valencia, España) y la filósofa, crítica de arte y escritora Amparo Zacarés Pamblanco (Valencia, España). Recientemente se han incorporado a la Colectiva Portal de Igualdad Yolanda Herranz Pascual, artista y catedrática de escultura de la Universidad de Vigo (Baracaldo-Vizcaya, España) y Rosa Mascarell Dauder, artista, comisaria y gestora cultural (Gandía-Valencia, España). visuales. Fue creado en diciembre de 2020 siguiendo la iniciativa del movimiento transnacional de mujeres WAM MUSEUM en su compromiso con el arte contemporáneo y con la equidad. Para tal ocasión, se instaló una lona en el Ágora de la UPV que invitaba a participar a toda la comunidad universitaria. Esta intervención pública, ideada y diseñada respectivamente por las artistas feministas Mau Monleón Pradas y María Penalva-Leal, se difundió a través de las redes sociales a nivel global, principalmente en América latina: Brasil, Argentina, Chile y México. Hay que recalcar que se trata de un museo virtual, creado por mujeres y hombres, para visibilizar la obra de las mujeres y que está abierto a la libre participación de todas las personas que quieran subir una o varias obras de mujeres artistas. Se procede subiendo una imagen o video a instagram, facebook, o twitter, añadiendo el hasthag y etiqueta del Women Artist Museum así como la web y las redes de las artistas. Para su realización, se convocó una acción masiva el 29 de diciembre de 2020 y se llegaron a reportar varios centenares de publicaciones internacionales en menos de un mes con el hashtag \#womenartistmuseumwam. Sigue activo y pueden seguir realizándose aportaciones ${ }^{4}$.

Entre otras más, también podrían citarse las acciones relacionadas con la participación de la Colectiva en foros universitarios y medios de comunicación, como la conferencia que en el mes de febrero de 2021 impartió en el IVAM la artista, que estuvo acompañada por varios componente de la Colectiva que tuvieron voz y palabra en el acto. En este capítulo también es digno de mención el haberse interesado en dejar constancia por escrito del planteamiento y de su metodología trasmedia (Zacarés y Monleón Pradas, 2021) o el haber colaborado en el Proyecto: «Las Muje-

4 Vid. https://mailchi.mp/a8ff22b29c/women-artist-museum 
res cambian los Museos. De la igualdad a la equidad», que tuvo lugar en junio de $2021 \mathrm{y}$ que se desarrolló desde la plataforma online de la Universidad Complutense de Madrid, en unión con la Universidad de Buenos Aires y la Universidad de Sao Paulo de Brasil. Por el momento, cuando aún no hace ni un año que se inició la Campaña de arte público trasmedia y feminista \#PortaldeIgualdad, puede decirse que el balance es muy positivo, ya que se ha internacionalizado la campaña y se ha dado impulso a la corriente de opinión que demanda justicia de género e igualdad de oportunidades para las mujeres artistas. En suma, la Colectiva, en su esfuerzo por conseguir una sociedad más justa e igualitaria a través de un proyecto artístico que se concibe como un work in progress, destaca por su carácter activista y por saber conjugar la estética y la ética para lograr, dentro del sistema del arte, un cambio de paradigma que vaya dirigido a la transformación de los museos y los centros de arte.

\section{VI}

En cualquier caso, aún queda mucho por conseguir, máxime cuando no bastan las declaraciones formales y lo que se demanda es que la igualdad sea real y efectiva. Sin embargo, en los últimos años se están abriendo diversos cauces para que los museos caminen hacia la equidad. Un ejemplo cercano es el proyecto $R e$ lecturas. Itinerarios Museales en Clave de género, subvencionado por la Generalitat Valenciana a partir de un estudio de la Universitat de València. Se trata de un proyecto donde varios museos valencianos presentan sus colecciones desde una perspectiva de género, invitando al público a reflexionar sobre los mitos patriarcales y la desigualdad estructural con la que históricamente se han diferenciado los roles y las funciones entre los sexos. En esta iniciativa participan diversas tipologías de museos como

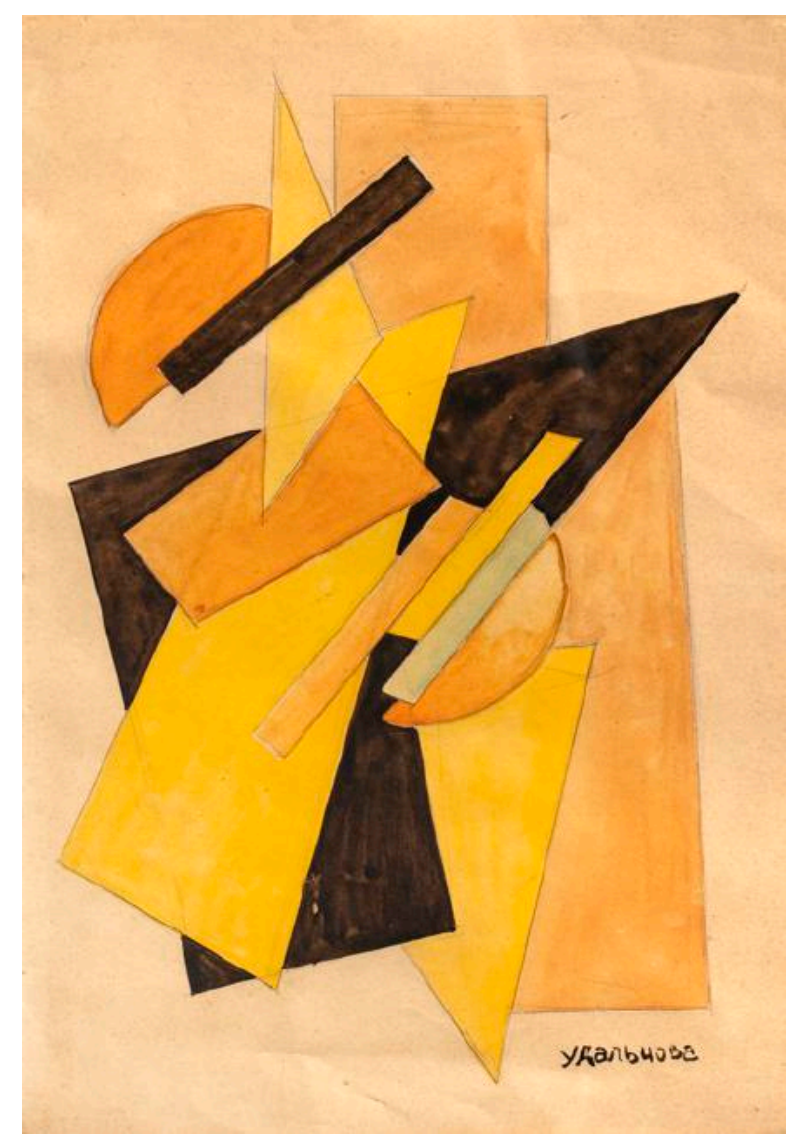

Acuarela y lápiz sobre papel de Nadezhda Udaltsova de 1916, actualmente en el Museu d'Art Contemporani Vicente Aguilera Cerni de Vilafamés procedente de la colección Fracaral.

son, por ejemplo, el Museo Nacional de Cerámica y Artes Suntuarias González Martí, el Museu de Etnología o el Museu de Belles Arts de València, y así hasta un total de 15 museos $^{5}$.

En este apartado, hay que reseñar la labor realizada en el Museu d'Art Contemporani Vicente Aguilera Cerni de Vilafamés (MACVAC) a través de la gestión de su actual directora Rosalía Torrent, quien también forma parte de la Colectiva Portal de Igualdad. Al respecto, conviene recordar que el museo y todo el personal que trabaja en él, acogió las indicaciones de equidad de género desde el momento inicial en que se presentó el Manifiesto Portal de Igualdad para Museos y Centros de Arte. Además, reciente-

5 Vid. http://relecturas.es/proyecto/ [Fecha de consulta 26/7/2021] 
mente, al recibir una serie de obras procedente de la colección Fracaral, se ha podido constatar con cuánta obra de mujeres artistas contaba. Lo cierto es que no eran muchas pero lo determinante es que, ante este desequilibrio, el propio coleccionista ha asumido la importancia de adquirir más trabajos de autoría femenina.

Finalmente, conviene señalar que la Comunitat Valenciana cuenta desde hace unos años con un catálogo de mujeres artistas (Aguilar Civera, 2001) que, en su mayoría, no han sido debidamente reconocidas dado el sesgo androcéntrico y sexista que ha dominado la historia del arte. Ahora bien, siendo obvio que todavía se privilegia antes al varón que a la mujer, lo cierto es que ya comienza a hacerse patente el agotamiento histórico del paradigma patriarcal en la cultura. Un proceso que puede detectarse desde la última década del siglo pasado hasta nuestros días, cuando muchas de las aportaciones artísticas de las mujeres cuestionaron un modelo único de feminidad y trataron, desde una óptica feminista, problemáticas que procedían de sus experiencias vitales y que se derivaban del mismo hecho de ser mujer. En su diversidad, podría decirse que sus poéticas se inscribían dentro de una estética de disidencia que consideraba urgente incluir la perspectiva de género en los museos, galerías y otros espacios artísticos. Por este motivo, no resulta baladí que la Generalitat Valenciana, en sus cuatro últimas convocatorias públicas, venga seleccionando obra de mujeres artistas para su colección de arte contemporáneo. En esa línea, los trabajos se adquieren con el fin de completar el patrimonio cultural y ampliar los fondos museísticos. Pero, sobre todo, esas adquisiciones se realizan en base a criterios de calidad y de equidad, asumiendo los códigos de buenas prácticas (Silvestre, 2018: 27). Es desde este planteamiento, cuando las obras acometidas por las mujeres artistas entran en los museos por derecho propio y caben, de manera legítima, sus reivindicaciones sociales, culturales y políticas.
Aún queda mucho por recórrer, pero ya está puesta la senda por la que los museos pueden transitar si quieren ofrecer una visión más holista de la realidad y contribuir a la democratización real del mundo del arte. Tomemos como referencia las iniciativas que hemos citado como son la campaña de arte público feminista \#PortaldeIgualdad y la Herramienta de Autodiagnóstico MAV para la Igualdad. Por eso mismo, sería interesante preguntarse ¿qué ocurriría si los Museos y Centros de Arte aceptaran sin dilación las sugerencias y las propuestas de mejora tras contestar el cuestionario de autodiagnóstico MAV? O también, ¿qué pasaría si los puntos programáticos del Manifiesto Portal de Igualdad tuvieran concreción real en los Museos y Centros de Arte? El panorama, desde luego, sería bien distinto al actual pues, tanto a nivel nacional como internacional, habrían quedado desactivadas todas las objecciones y los prejuicios que durante siglos circularon sobre la valía de las mujeres artistas en su condición de sujeto creador. Tampoco habría que sugerir que en los espacios diferenciados de las ferias, las galerías expusieran obra realizada por mujeres para darles visibilidad, ni habría que motivar al Consejo Internacional de Museos (ICOM) para que las mujeres artistas tuvieran presencia en el Día Internacional de los Museos (DIM). Todo sería bien diferente, la gestión museística sería más justa y democrática y la historia del arte, por fin, habría quedado reelaborada desde parámetros equitativos.

\section{REFERENCIAS Y BIBLIOGRAFÍA}

Aguilar Civera, Inmaculada (2001) Arte Valenciano contemporáneo. Una recopilación bibliográfica desde 1976, Valencia: Generalitat Valenciana.

Arribas Pérez, Carmelo (2007) Ilustres y desconocidas, Instituto de la Mujer de la Junta de Extremadura. 
BASElitz, Georg (2013) «Interview with German Painter», Der Spiegel. Disponible en https://www.spiegel. de/international/germany/spiegel-interview-with-german-painter-georg-baselitz-a-879397.html [Fecha de consulta 21/7/2021]

Clark, Nick (2013) «What's the biggest problem with women artists?», The Independent, 16/2/2013.

Domínguez, Yolanda (2018) «\#estamos aquí». Disponible en https://www.youtube.com/watch?$\mathrm{v}=\mathrm{g}-\mathrm{N} 5 \mathrm{BOMrK3}$ [Fecha de consulta 21/7/2021]

Feliu, Joan (2019) «Ausencias invisibles. Mujeres artistas en las ferias de arte contemporáneo», Dossiers feministes, 23:111-124.

Guerrilla Girls (1985) «Code of ethics for Art Museums», en Guerrilla Girls 1985-2013, Catálogo (2013), Bilbao: Centro de Arte y Cultura Alhóndiga.

- (1988) «The Advantages of being a Woman Artist», en Guerrilla Girls 1985-2013, Catálogo (2013), Bilbao: Centro de Arte y Cultura Alhóndiga.

Gimeno, María (2020) «Queridas Viejas Project» Consejería de cultura. Filmoteca de Cantabria 2020. Fotografía de Ana Martín@. Disponible en https:// WWw.mariagimeno.com/QUERDAS-VIEJAS-PROJECT [Fecha de consulta 21/7/2021]

Halperin, Julia \& Charlotte Burns (2019) «Female Artists Represent Just 2 Percent of the Market. Here's Why-and How That Can Change», en Arnet News. Disponible en https://news.artnet.com/ womens-place-in-the-art-world/female-artists-represent-jus-2-percent-market-heres-can-change-1654954.

LipPARD, Lucy (1976) From the Center. Feminist Essays on Women's Art. New York: Dutton.

López Fernández-CaO, Marián (2000) «La creación artística: un difícil sustantivo femenino», en Marián López Fernández Cao (coordinadora) Creación artística y mujeres. Recuperar la memoria, Madrid: Narcea.

- (2008) «Algunas consideraciones sobre la capacidad de vivir en equidad. Propuestas desde la creación», en Huerta, Ricard y Romà de la CAlle (eds.) Mentes sensible. Investigar en educación y museos, Valencia: PUV.

López Fernández-CaO, Marián y Alma Portal
LLEDó (2020) «Autodiagnóstico MAV para la Igualdad en los Museos y Centros de Arte». Disponible en https://mav.org.es/wp-content/ uploads/2020/12/Fundamentaci\%C3\%B3nAutodiagn\%C3\%B3stico-MAV.pdf [Fecha de consulta 22/7/2021]

Monleón-Pradas, Mau (2017) Women in work. Mujer arte y trabajo en la globalización. Catálogo de la exposición en la Universidad Politécnica de Valencia.

Nochlin, Linda (1971) «Why there have not been great women artists?», en Women, Art and Power and other essays, London: Thames and Hudson.

PADró, Carla (2003) «La museología crítica como una forma de reflexionar sobre los museos como zonas de conflicto e intercambio», en LoRente, Jesús Pedro (dir.) Museología crítica y Arte contemporáneo, Zaragoza: Prensas Universitarias.

Riaño, Peio H. (2021) «Las galerías más caras no quieren mujeres artistas (en Arco tampoco)» https:// $w w w$.eldiario.es/cultura/arte/galerias-caras-no-quieren-mujeres-artistas-arco_1_8110305.html [Fecha de consulta 21/7/2021]

Roberts, Lisa C. (1997) From Knowledge to narrative. Educators and the changing museum, Washington: Smithsonian Institution Press.

Silvestre, Ricard (2018) «Col.lecció d'art contemporani de la Generalitat Valenciana. Incerteses i divergències», en Art Contemporani de la Generalitat Valenciana/Primers Moments, València: GV/cccC Consorci de Museus de la Comunitat Valenciana.

ZaCarés, Amparo y Mau Monleón-Pradas (2021) «Arte público activista. Estrategias participativas y trasmedia en el proyecto feminista \#PortaldeIgualdad dirigido a la transformación de los museos y los centros de arte» en ANIAV, Revista de Investigación en Artes Visuales de la UPV, 08. Disponible en http:// hdl.handle.net/10251/165177 [Fecha de consulta 27/7/2021]

Recibido el 25 del 8 de 2021

Aceptado el 22 del 9 de 2021 BIBLID [2530-1330 (2021): 28-45] 